\title{
DIVISIBLE MODULES
}

\section{EBEN MATLIS}

Introduction. Let $R$ be an integral domain with quotient field $Q$, and let $A$ be a module over $R$. $A$ is said to be a divisible $R$-module, if $r A=A$ for every $r \neq 0 \in R$. An element $x \in A$ is said to be a torsion element of $A$, if there exists $r \neq 0 \in R$ such that $r x=0$. The set of torsion elements of $A$ is a submodule of $A$ called the torsion submodule of $A$, and we will consistently denote it by $A_{T}$. We will let $E(A)$ denote the injective envelope of $A$ (see [3]); and hd ${ }_{R} A$ will denote the homological dimension of $A$ as an $R$-module.

We will study conditions, some necessary, some sufficient, for the torsion submodule of a divisible module to be a direct summand. These will be related to the condition that $\operatorname{hd}_{R} Q=1$, where $Q$ is the quotient field of $R$. We will apply these conditions to show that, if $R$ is a Noetherian integral domain in which prime ideals different from zero are maximal, and if $D$ is a divisible module over $R$, then $D$ is a homomorphic image of an injective $R$-module and $D_{T}$ is a direct summand of $D$. The same conclusions hold, if we merely assume for an arbitrary integral domain that its quotient field is countably generated as a module over the ring.

1. The torsion submodule. It is easy to see that, if $C$ is an injective module over an integral domain $R$, then its torsion submodule $C_{T}$ is also an injective $R$-module, and therefore a direct summand. Namely, any homomorphism of an ideal of $R$ into $C_{T}$ can be extended to a homomorphism of $R$ into $C$; but this extension must in fact map $R$ into $C_{T}$. The following theorem is a generalization of this fact.

TheOREM 1.1. Let $R$ be an integral domain and $H$ a homomorphic image of an injective $R$-module. Then $H_{T}$ is a direct summand of $H$.

Proof. The mapping of a free $R$-module $F$ onto an injective $R$ module $C$ can be extended to a mapping of $E(F)$ onto $C$. We can thus assume that there exists a torsion-free, divisible $R$-module $U$ and an epimorphism $f: U \rightarrow H$. Now $H / H_{T}$, being torsion-free and divisible, is a direct sum of $R$-modules $Q_{i}$, where $Q_{i}$ is isomorphic to $Q$ the quotient field of $R$. Let $S_{i}$ be the inverse image of $Q_{i}$ under the canonical map $H \rightarrow H / H_{T}$. We will prove that $H_{T}$ is a direct summand of each $S_{i}$, and by [2, Lemma 2] this will complete the proof of the theorem.

Presented to the Society, September 3, 1959; received by the editors May 23, 1959. 
Let $y \in S_{j}-H_{T}$; then $R y$ is a torsion-free submodule of $S_{j}$. Choose $x \in U$ such that $f(x)=y$. Since $U$ is a vector space over $Q$, there exists an $R$-submodule $T_{j}$ of $U$ such that $T_{j} \cong Q$ and $x \in T_{j}$. If $u \neq 0 \in T_{j}$, there exist $r, s \in R$ such that $r u=s x \neq 0$. Since $r f(u)=s f(x)=s y \neq 0$, we have $f(u) \neq 0$, and thus $f\left(T_{j}\right) \cong T_{j} \cong Q$.

Since $H=\sum_{i} S_{i}, f(u)=w+z$, where $w \in \sum_{i \neq j} S_{i}$ and $z \in S_{j}$. Hence $r w+r z=r f(u)=s y \in S_{j}$. Thus $r w \in \sum_{i \neq j} S_{i} \cap S_{j}=H_{T}$. Therefore, $w \in H_{T} \subset S_{j}$, and so $f(u) \in S_{j}$. This shows that $f\left(T_{j}\right) \subset S_{j}$. Since $f\left(T_{j}\right) \cong Q$, we have $f\left(T_{j}\right) \cap H_{T}=0$ and $f\left(T_{j}\right)$ maps onto $Q_{j}$ under the canonical map $H \rightarrow H / H_{T}$. Thus $S_{j}=H_{T} \oplus f\left(T_{j}\right)$; and so $H_{T}$ is a direct summand of $H$.

Theorem 1.2. Let $R$ be an integral domain with quotient field $Q \neq R$. Suppose that $D_{T}$ is a direct summand of $D$ for every divisible $R$-module $D$. Then $\operatorname{hd}_{R} Q=1$.

Proof. Let $A$ be any $R$-module and let $E=E(A)$. Since $E$ is an essential extension of $A, E / A$ is a torsion $R$-module. Let $G$ be any $R$-module extension of $E / A$ by $Q$. Since both $Q$ and $E / A$ are divisible, $G$ is also divisible. Clearly $E / A$ is the torsion submodule of $G$; and thus by assumption $E / A$ is a direct summand of $G$. Thus $\operatorname{Ext}_{R}^{1}(Q, E / A)$ $=0\left[1\right.$, Theorem 14.1.1]. We also have $\operatorname{Ext}_{R}^{n}(Q, E)=0$ for $n>0$, since $E$ is injective. Therefore, from the exact sequence:

$$
\operatorname{Ext}_{R}^{1}(Q, E / A) \rightarrow \operatorname{Ext}_{R}^{2}(Q, A) \rightarrow \operatorname{Ext}_{R}^{2}(Q, E)
$$

we deduce that $\operatorname{Ext}_{R}^{2}(Q, A)=0$. Hence $\operatorname{hd}_{R} Q \leqq 1$. Since $Q$ is not $R$-projective, $\operatorname{hd}_{R} Q=1$.

Over an arbitrary integral domain it is not true that $D_{T}$ is a direct summand for every divisible module $D$. For by the above theorem this would imply that $\operatorname{hd}_{R} Q=1$. However, I. Kaplansky has shown (unpublished) that $\operatorname{hd}_{R} Q=1$ for a valuation ring $R$ if and only if $Q$ is a countably generated $R$-module.

Theorem 1.3. Let $R$ be an integral domain with quotient field $Q \neq R$, and suppose that $Q$ is countably generated as a $R$-module. Then every divisible $R$-module $D$ is a homomorphic image of an injective $R$-module. Thus $D_{T}$ is a direct summand of $D$, and $\operatorname{hd}_{R} Q=1$.

Proof. There exists a countable set of generators $\left\{q_{n}\right\}$ for $Q$ over $R$, and elements $\left\{a_{n+1}\right\}$ of $R$ such that $q_{1}=1$ and $a_{n+1} q_{n+1}=q_{n}$. Let $D$ be a divisible $R$-module, and let $x \neq 0 \in D$. We define a mapping $f$ from the generators $\left\{q_{n}\right\}$ to $D$. Let $f(1)=x$; then there exists $x_{2} \in D$ such that $a_{2} x_{2}=x$, and we define $f\left(q_{2}\right)=x_{2}$. There exists $x_{3} \in D$ such that $a_{3} x_{3}=x_{2}$, and we define $f\left(q_{3}\right)=x_{3}$. We continue in this way and 
define $f$ on all the generators $\left\{q_{n}\right\}$. It is easily verified that $f$ induces an $R$-homomorphism from $Q$ into $D$ such that the image contains $x$. It is now clear that by taking a big enough direct sum $G$ of copies of $Q$ we can define an $R$-homomorphism of $G$ onto $D$.

It should be remarked that if $R$ is any integral domain and $S$ a countable, multiplicatively closed subset of $R$, then it can be easily shown that if $F$ is a countably generated free $R$-module and $f$ a suitably chosen mapping of $F$ onto $R_{S}$, then the kernel of $f$ is free; and thus $\mathrm{hd}_{R} R_{S} \leqq 1$.

2. $\operatorname{hd}_{R} Q=1$.

Proposition 2.1. Let $R$ be an integral domain with quotient field $Q$ such that $\operatorname{hd}_{R} Q=1$. Let $H$ be an $R$-module. Then the following statements are equivalent:

(1) $\operatorname{Ext}_{R}^{1}(Q / R, H)=0$.

(2) Every $R$-homomorphism from $R$ into $H$ can be extended to an $R$-homomorphism from $Q$ into $H$.

(3) $H$ is a homomorphic image of an injective $R$-module.

Proof. That (1) implies (2) follows immediately from the exact sequence:

$$
\operatorname{Hom}_{R}(Q, H) \rightarrow \operatorname{Hom}_{R}(R, B) \rightarrow \operatorname{Ext}_{R}^{1}(Q / R, H) .
$$

That (2) implies (3) is trivial. That (3) implies (1) follows from the fact that $\operatorname{hd}_{R} Q / R=1$.

Proposition 2.2. Let $R$ be an integral domain with quotient field $Q$ such that $\operatorname{hd}_{R} Q=1$. Let $H$ be an $R$-module. Then:

(1) If $H$ is a homomorphic image of an injective $R$-module, so is $H_{T}$.

(2) If $B$ is a submodule of $H$ and if $B$ and $H / B$ are homomorphic images of injective modules, then so is $H$.

Proof.

(1) If $H$ is a homomorphic image of an injective $R$-module, then $H_{T}$ is a direct summand of $H$ by Theorem 1.1. Hence $H_{T}$ is also a homomorphic image of an injective $R$-module.

(2) Suppose that $B$ and $H / B$ are homomorphic images of injective $R$-modules. We have an exact sequence:

$$
\operatorname{Ext}_{R}^{1}(Q / R, B) \rightarrow \operatorname{Ext}_{R}^{1}(Q / R, H) \rightarrow \operatorname{Ext}_{R}^{1}(Q / R, H / B) .
$$

By Proposition 2.1 the two end modules are zero, and thus $\operatorname{Ext}_{R}^{1}(Q / R, H)=0$. Hence by Proposition 2.1 again, $H$ is a homomorphic image of an injective $R$-module. 
Definition. Let $B$ be a module over an integral domain. Then we will say that $B$ is h-reduced, if $B$ has no nonzero submodules which are homomorphic images of injective modules.

Corollary 2.3. Let $A$ be a module over an integral domain $R$ with quotient field $Q$ such that $\operatorname{hd}_{R} Q=1$. Then $A$ has a unique largest submodule $H$ which is a homomorphic image of an injective $R$-module, and $A / H$ is h-reduced.

Proof. Let $H$ be the sum of all submodules of $A$ which are homomorphic images of injective $R$-modules. It is clear that $H$ is the unique largest submodule of $A$ which is a homomorphic image of an injective $R$-module. Suppose that $B / H$ is a homomorphic image of an injective $R$-module, where $B$ is a submodule of $A$ containing $H$. Then by Proposition $2.2 B$ is a homomorphic image of an injective $R$-module. Therefore, $B=H$ and $B / H=0$.

Proposition 2.4. Let $R$ be an integral domain with quotient field $Q$ such that $\operatorname{hd}_{R} Q=1$. Let $D$ be a divisible module over $R$, and let $H$ be $a$ submodule of $D_{T}$ such that $H$ is a homomorphic image of an injective $R$-module. Then $D_{T}$ is a direct summand of $D$ if and only if $D_{T} / H$ is a direct summand of $D / H$.

Proof. Suppose that $D_{T}$ is a direct summand of $D$, and let $S$ be a complementary summand of $D_{T}$ in $D$. Then $D / H \cong D_{T} / H \oplus S$, and since $D_{T} / H$ is the torsion submodule of $D / H, D_{T} / H$ is a direct summand of $D / H$. Conversely, suppose that $D / H=D_{T} / H \oplus G / H$, where $G$ is a submodule of $D$ containing $H$. Now $G / H$ is torsion-free and divisible, hence injective. Thus by Proposition 2.2 and Theorem 1.1 $H$ is a direct summand of $G$. Let $L$ be a complementary summand of $H$ in $G$. Then it is clear that $D=D_{T} \oplus L$.

Proposition 2.5. Let $R$ be an integral domain with quotient field $Q$, and let $T$ be an h-reduced torsion $R$-module. Then $\operatorname{Ext}_{R}^{1}(Q, T)=0$ if and only if $T \cong \operatorname{Ext}_{R}^{1}(Q / R, T)$.

Proof. Since $\operatorname{Hom}_{R}(Q, T)=0$, we have an exact sequence:

$$
0 \rightarrow \operatorname{Hom}_{R}(R, T) \rightarrow \operatorname{Ext}_{R}^{1}(Q / R, T) \rightarrow \operatorname{Ext}_{R}^{1}(Q, T) \rightarrow 0 .
$$

It follows that if $\operatorname{Ext}_{R}^{1}(Q, T)=0$, then $T \cong \operatorname{Ext}_{R}^{1}(Q / R, T)$. Conversely, if $T \cong \operatorname{Ext}_{R}^{1}(Q / R, T)$, then the above exact sequence shows that $\operatorname{Ext}_{R}^{1}(Q, T)$ is a torsion module. However, $\operatorname{Ext}_{R}^{1}(Q, T)$ is torsion-free, and thus $\operatorname{Ext}_{R}^{1}(Q, T)=0$.

Corollary 2.6. Let $R$ be an integral domain with quotient field $Q$. 
Then the torsion submodule of a divisible $R$-module is always a direct summand if and only if the following two conditions hold:

(1) $\operatorname{hd}_{R} Q=1$.

(2) $T \cong \operatorname{Ext}_{R}^{1}(Q / R, T)$, whenever $T$ is an h-reduced, torsion, divisible $R$-module.

Proof. The necessity follows from Theorem 1.2 and Proposition 2.5; the sufficiency follows from Corollary 2.3 and Propositions 2.4 and 2.5 .

Proposition 2.7. Let $R$ be an integral domain with quotient field $Q$ such that $\operatorname{hd}_{R} Q=1$ and gl. $\operatorname{dim} . R \leqq 2$. Let $S$ be any torsion-free $R$ module. Then $\operatorname{hd}_{R} S \leqq 1$. Thus if $A$ is an $R$-module such that $A_{T}$ is a homomorphic image of an injective $R$-module, then $A_{T}$ is a direct summand of $A$.

Proof. Let $B$ be any $R$-module. Then from the exact sequence:

$$
0 \rightarrow S \rightarrow Q \otimes_{R} S \rightarrow Q / R \otimes_{R} S \rightarrow 0
$$

we derive the exact sequence:

$$
\operatorname{Ext}_{R}^{2}\left(Q \otimes_{R} S, B\right) \rightarrow \operatorname{Ext}_{R}^{2}(S, B) \rightarrow \operatorname{Ext}_{R}^{3}\left(Q / R \otimes_{R} S, B\right) .
$$

Since $\operatorname{hd}_{R} Q=1$ and gl. $\operatorname{dim} . R \leqq 2$, the two end modules are zero Thus $\operatorname{hd}_{R} S \leqq 1$, and the rest of the theorem follows immediately.

\section{Krull dimension $=1$.}

Throughout this section $R$ will be a Noetherian integral domain with the property that nonzero prime ideals are maximal. We will let $Q$ be the quotient field of $R$ and $K=Q / R$.

Definition. Let $A$ be an $R$-module and $M$ a prime ideal of $R$. We will say that $A$ is $M$-primary, if for any $x \neq 0 \in A$, the order ideal of $x$ is an $M$-primary ideal. If $B$ is any $R$-module, and $A$ is the set of all elements of $B$ whose order ideal is $M$-primary (together with the element 0 ), then $A$ is an $M$-primary $R$-module which we will call the $M$-primary component of $B$.

Lemma 3.1. Let $B$ be any torsion $R$-module. Then $B$ is the direct sum of its $M$-primary components, $M$ ranging over the prime ideals of $R$. Furthermore, $B \otimes_{R} R_{M}$ is the M-primary component of $B$.

Proof. Let $\left\{M_{a}\right\}$ be the collection of nonzero prime ideals of $R$. By [3, Theorem 3.3] $E(B)=\sum_{a} \oplus E_{a}$, where $E_{a}$ is the $M_{a}$-component of $E(B)$. Let $B_{a}=B \cap E_{a}$; then $B_{a}$ is the $M_{a}$-component of $B$. Let $x \neq 0 \in B$; then $x=x_{1}+\cdots+x_{n}$, where $x_{i} \in E_{i}$. Now $\bigcap_{i=2}^{n} M_{i} \llbracket M_{1}$; 
hence there exists $s \in \bigcap_{i-\infty}^{n} \quad M_{i}$ such that $s \notin M_{1}$. Then there exists an integer $k>0$ such that $s^{k} x_{i}=0$ for $i=2, \cdots, n$. Hence $s^{k} x=s^{k} x_{1}$. There are elements $m \in M$ and $t \in R$ such that $1=m+t s^{k}$. There is an integer $q>0$ such that $m^{q} x_{1}=0$. Since $1=m^{q}+r s^{k}, r \in R$, we have $x_{1}=r s^{k} x_{1}=r s^{k} x \in B_{1}$. Similarly $x_{i} \in B_{i}$ for $i=2, \cdots, n$. Thus $B=\sum_{a} \oplus B_{a}$.

Let $M_{v}$ be a prime ideal of $R$. Clearly $B_{a} \otimes_{R} R_{M_{v}}=0$, if $M_{a} \neq M_{v}$. Thus $B \otimes_{R} R_{M_{v}}=B_{v} \otimes_{R} R_{M_{v}}$. It is easily seen that the canonical map $B_{v} \rightarrow B_{v} \otimes_{R} R_{M_{v}}$ is an epimorphism. However, since $B_{v}$ is $M_{v}$-primary, the kernel of this map is zero. Thus $B_{v}=B_{v} \otimes_{R} R_{M_{v}}$, and so $B \otimes_{R} R_{M_{v}}$ $=B_{v}$.

Lemma 3.2. $\operatorname{hd}_{R} Q=1$.

Proof. It is sufficient to prove that $\mathrm{hd}_{R} K=1$. By Lemma 3.1 $K=\sum_{a} \oplus K_{M_{a}}$; thus it is sufficient to prove that $h_{R} K_{M_{v}}=1$. For this it is sufficient to prove that if $D$ is any divisible $R$-module, then $\operatorname{Ext}_{R}^{1}\left(K_{M_{v}}, D\right)=0$. Let $A$ be any extension of $D$ by $K_{M_{v}}$; then $A$ is a divisible $R$-module. We have $D=\sum_{a} \oplus D_{M_{a}}$ and $A=\sum_{a} \oplus A_{M_{a}}$. Clearly $D_{M_{a}}=D \cap A_{M_{a}}$. Hence for $a \neq v$, we have $D_{M_{a}}=A_{M_{a}}$. Thus we have an exact sequence:

$$
0 \rightarrow D_{M_{v}} \rightarrow A_{M_{v}} \rightarrow K_{M_{v}} \rightarrow 0,
$$

and all of the modules and mappings of this sequence are $R_{M_{v}}$ modules and mappings. Thus we can assume that $R$ is a local ring with a single nonzero prime ideal $M$.

Take $s \neq 0 \in M$, and let $S$ be the multiplicatively closed set consisting of the powers of $s$. Now $R_{S} \subset Q$; on the other hand, the prime ideals of $R_{S}$ and the prime ideals of $R$ not meeting $S$ are in 1-1 correspondence. Thus $R_{S}$ is a field, and $R_{S}=Q$. Therefore, $Q$ is a countably generated $R$-module; and thus $\operatorname{hd}_{R} Q=1$ by Theorem 1.3 , or the remark following it.

Theorem 3.3. Every divisible $R$-module $D$ is a homomorphic image of an injective $R$-module; and thus $D_{T}$ is a direct summand of $D$.

Proof. Since hd ${ }_{R} Q=1$ by Lemma 3.2, it follows from Proposition 2.2 that we only need to prove that $D_{T}$ is a homomorphic image of an injective $R$-module. By Lemma 3.1 $D_{T}=\sum_{a} \oplus D_{T_{a}}$, where $D_{T_{a}}$ $=D_{T} \otimes_{R} R_{M_{a}}$ is a divisible $R_{M_{a}}$-module. As we have seen in Lemma 3.2, $Q$ is a countably generated $R_{M_{a}}$-module; and thus by Theorem $1.3, D_{T_{a}}$ is a homomorphic image of an injective $R_{M_{a}}$-module. Thus $D_{r_{\mathrm{a}}}$ is a homomorphic image of an injective $R$-module; and therefore, the same is true of $D_{T}$. 


\section{REFERENCES}

1. H. Cartan and S. Eilenberg, Homological algebra, Princeton University Press, 1956.

2. I. Kaplansky, Modules over Dedekind rings and valuation rings, Trans. Amer. Math. Soc. vol. 72 (1952) pp. 327-340.

3. E. Matlis, Injective modules over Noetherian rings, Pacific J. Math. vol. 8 (1958) pp. 511-528.

NORTHWESTERN UNIVERSITY

\section{A CHARACTERIZATION OF ALGEBRAIC NUMBER FIELDS WITH CLASS NUMBER TWO ${ }^{1}$}

\section{CARLITZ}

Let $Z=R(\theta)$ denote an algebraic number field over the rationals with class number $h$. It is familiar that $h=1$ if and only if unique factorization into prime holds for the integers of $Z$. For fields with $h \leqq 2$ we have the following criterion.

THEOREM. The algebraic number field $Z$ has class number $\leqq 2$ if and only if for every nonzero integer $\alpha \in Z$ the number of primes $\pi_{j}$ in every factorization

$$
\alpha=\pi_{1} \pi_{2} \cdots \pi_{k}
$$

depends only on $\alpha$.

Suppose first that $h=2$ and consider the factorization into prime ideals

$$
(\alpha)=\mathfrak{p}_{1} \cdots \mathfrak{p}_{s} \mathfrak{r}_{1} \cdots \mathfrak{r}_{t},
$$

where the $\mathfrak{p}_{j}$ are principal ideals while the $\mathfrak{r}_{j}$ are not. Then

$$
p_{j}=\left(\pi_{j}\right) \quad(j=1, \cdots, s) .
$$

Since $h=2$, it follows that

$$
\mathfrak{r}_{i} \mathfrak{r}_{j}=\left(\rho_{i j}\right) \quad(i, j=1, \cdots, t) ;
$$

moreover $t$ must be even, $=2 u$, say. Thus every factorization into primes implied ". by (2), for example

Received by the editors August 3, 1959.

1 Research sponsored by National Science Foundation grant NSF G-9425. 